Scientific Visualization, 2019, volume 11, number 4, pages 102 - 114, DOI: 10.26583/sv.11.4.09

\title{
Generalized Computational Experiment and Visual Analysis of Multidimensional Data
}

\author{
A.E. Bondarev ${ }^{1, A}$, V.A. Galaktionov ${ }^{2, A}$ \\ Keldysh Institute of Applied Mathematics Russian Academy of Sciences \\ ${ }^{1}$ ORCID: oooo-0003-3681-5212, bond@keldysh.ru \\ 2 ORCID: oooo-0001-6460-7539, vlgal@gin.keldysh.ru
}

\begin{abstract}
The work considers the problems of constructing a generalized computational experiment in the problems of computational aerodynamics. The construction of a generalized computational experiment is based on the possibility of carrying out parallel calculations of the same problem with different input data in multitasking mode. This allows carrying out parametric studies and solving problems of optimization analysis. The results of such an experiment are multidimensional arrays, for the study of which visual analytics methods should be used. The construction of a generalized experiment allows one to obtain dependences for valuable functionals on the determining parameters of the problem under consideration. The implementation of a generalized experiment allows one to obtain a solution for a class of problems in the ranges under consideration, and not just for one problem. Examples of constructing a generalized computational experiment for various classes of problems of computational aerodynamics are presented. The article also provides an example of constructing such an experiment for a comparative assessment of the accuracy of numerical methods. This approach is a synthesis of parallel computing, multi-dimensional data processing methods and visual analysis. The application of this approach makes it possible to increase the efficiency of research for a number of applied problems of mathematical modeling.
\end{abstract}

Keywords: generalized computational experiment, visual analysis, multidimensional data, parallel computations.

\section{Introduction}

The physical experiment was the main and often the only source of information on the problems of gas dynamics long before the advent of the computer age. In practice, the main goal of a physical experiment was almost always not to model the physical phenomenon itself, but to clarify the circumstances under which it occurs, i.e. obtaining the dependence of the appearance of the phenomenon on the determining parameters of the problem, such as Mach numbers, Reynolds numbers, Prandtl numbers, and the geometric parameters of the problem. Such large-scale experimental work made it possible to obtain key relationships for the dependence of the gasdynamic functions of interest or the conditions for the appearance of a physical effect on the key determining parameters. In fact, the establishment of such physical laws for shock waves, separated flows, characteristic configurations of streamlined bodies was the main task of fluid and gas mechanics.

As an example of such a dependence, one can cite the famous formula of G.I. Petrov, representing the fundamental law on the ultimate pressure drop in the shock, which the turbulent boundary layer is able to withstand without detachment from the wall [1]:

$$
P_{2} / P_{1}=0.713 M_{e}+0.213 \text {. }
$$

Here $P_{2} / P_{1}$ is the pressure drop, $M_{e}$ is the Mach number before the separation point, varying from 1.5 to 4 . 
Another example is the famous Kozlov formula [2], which represents the dependence of surface friction on Mach numbers, Reynolds numbers and the temperature factor:

$$
c_{f w}=0,085 R e_{w}^{-0,29+0,01 \lg R e_{w}} \bar{T}_{w e}^{0,39} \bar{T}_{e}^{0,2} .
$$

Here $c_{f w}, R e_{w}$ is the coefficient of surface friction and the Reynolds number calculated with reference to the wall temperature, $T_{e}$ is the temperature at the outer boundary of the boundary layer, and $T_{w e}$ is the temperature factor.

The advent of computer technology allowed solving the problems of mathematical modeling of currents, which sharply reduced the need for large-scale physical experiments. However, in the problems of mathematical modeling, the main tendency of carrying out series of calculations with the variation of the defining parameters of the problem also remained. The main goal was the same - to determine the conditions for the appearance of a physical phenomenon when the external conditions of the problem are varied. An example of such approach is described in article [8], which presents a series of numerical experiments on the flow of a backward ledge by a viscous gas flow. As a result of the experiments, a generalized formula is obtained that represents the characteristic time of the establishment of the flow as a function of the Mach and Reynolds numbers of the external flow.

Before the advent of parallel computing technologies, such calculations were difficult to implement and they were quite rare. However, they were the prototype of the generalized computational experiment.

The generalized computational experiment is based on solving direct and inverse problems of mathematical modeling. These tasks can be considered in a parametric and optimization setting. Problem solving is carried out using parallel technologies in multitasking mode. Numerical solutions are volumes of multidimensional data. To process and analyze this data, it is necessary to apply the methods of Data Analysis and Visual Analytics. The construction of a generalized computational experiment makes it possible to obtain a solution not for one, separately taken problem, but for a whole class of problems. The class of problems is determined in the ranges of variation of the defining parameters of the problem, such as characteristic numbers (Mach, Reynolds, Strouhal numbers, etc.) and geometric characteristics. In a practical sense, this makes it possible to reveal hidden dependences of valuable functionals on the determining parameters of the problem, similar to the above formulas. This work continues a series of works devoted to the development and implementation of a generalized computational experiment for various classes of computational aerodynamics problems [3-14].

Despite the fact that there are very few works devoted to the development of a generalized computational experiment, the development of such experiments is gradually taking place in many areas. First of all, tools are being developed to implement such experiments in many software packages for solving optimization problems. Here we can cite as an example the work [15], where algorithms are implemented that allow a generalized computational experiment in such fields as seismic exploration, plasma physics and turbid media optics, solving fundamental and applied problems of studying magnetic materials and creating spintronics devices, simulation of field development for the oil reservoir that contains kerogen with insitu combustion taken into account, simulation of poroelastic medium problems and hydraulic fracture problems.

\section{Prerequisites for the creation of a generalized compu- tational experiment}

The development of technologies and software tools for constructing a generalized computational experiment occurs as the modern development of mathematical methods and highperformance computing tools. Two main reasons should be pointed out as the main factors determining the possibility of efficiently constructing a generalized computational experiment.

The first of these is the emergence of high-performance computing clusters and parallel technologies. It is generally accepted that parallel technologies provide a) the ability of fast com- 
puting and b) the ability to use detailed computational grids. However, parallel technologies also provide researchers with another crucial opportunity. This is an opportunity of parallel calculation of the same problem with different input data in multitask mode. From the point of view of the author, this possibility is not yet fully appreciated. This possibility allows one to effectively solve parametric and optimization problems and construct a generalized computational experiment.

The second reason is the intensive development of methods and approaches for processing and visualization of multidimensional data. The results of a generalized computational experiment in the form of discrete multidimensional arrays need processing and analysis in order to obtain hidden interdependencies between the determining factors in the class of problems that interest the researcher.

\section{Generalized numerical experiment}

A generalized numerical experiment involves splitting each of the defining parameters of a problem within a certain range. A grid decomposition is formed for some multidimensional parallelepiped composed of the defining parameters of the considered problem of gas dynamics. For each point of this grid, the problem is calculated in the space of the determining parameters. According to [5, 9], this can be written as follows.

Suppose that there is a reliable numerical method for solving two-dimensional and threedimensional nonstationary problems of computational gas dynamics. Then we can obtain a numerical solution $F\left(x, y, z, t, A_{1}, \ldots, A_{N}\right)$ for any point in the space of a computational domain, where $x, y, z$ are the spatial coordinates, $t$ is the time, $A_{1}, \ldots, A_{N}$ are the defining parameters of the problem. As defining parameters of the problem, we will keep in mind the characteristic numbers describing the properties of the flow under consideration, such as the Mach numbers, Reynolds, Prandtl, Strouhal, etc., and the characteristic geometric parameters. Each of the characteristic parameters is limited in a certain range

$$
A_{i}^{\min } \leq A_{i} \leq A_{i}^{\max }, \quad i=1, \ldots, N .
$$

We divide each of the parameters $A_{i}$ into $k-1$ parts, so we obtain for each parameter a partition consisting of $k$ points. The volume of an $N$-dimensional space formed by a set of defining parameters $A_{i}$ is filled with a set of $k^{N}$ points.

Denoting the point from the given set, as $\left(A_{1}^{*}, \ldots, A_{N}^{*}\right)$, we arrive at the fact that for each point of the collection it is necessary to obtain a numerical solution of the gas-dynamic problem $F\left(, x, y, z, t, A_{1}^{*}, \ldots, A_{N}^{*}\right)$.

It is easy to see that this will require solving $k^{N}$ gasdynamic problems, which is impossible without the use of parallel calculations in a multitask mode. In practice, the number $N$ usually does not exceed 5, which corresponds to the computing capabilities at the current time.

It should also be noted that we formulated the classical problem of parametric study. Parametric numerical studies allow one to obtain a solution not for one particular mathematical modeling problem, but for a class of problems defined in a multidimensional space of defining parameters. Also, such a formal formulation allows numerical study of optimization analysis problems, when the inverse problem is solved at each point of the grid partition of the multidimensional space of the determining parameters. Both types of similar problems are considered in a series of papers [3-14].

The only way to effectively carry out a generalized numerical experiment is applying of parallel computations. The problem of the optimal and effective way of parallelization was thoroughly discussed in the papers $[7,8]$. There were considered parts of the whole algorithm for parameter optimization and analysis. For these parts the main criterion of applicability for parallelizing is independence of specific numerical method. From this point of view the most perspective way for parallelizing is applying the approach of multitask parallelism using the principle "one task - one process". Due to minimal quantity of internal exchanges between the processes we are able to create an effective practical tool for generalized numerical experiment. We assume that $k$ processes are provided for parallel computation. The control process 
$P_{o}$ creates the grid in the multidimensional space of determining parameters, then $P_{o}$ forms tasks and sends the tasks to others processes and to itself also. After task completion $P_{o}$ collects the results and implements all procedures defined by user, such as data processing and transformation. Due to the absence of internal exchanges between the processes the procedure of parallelizing amounts to creation of control interface for tasks distribution and data collecting in one multidimensional array.

There are some effective and easy ways to create such interface for parallel computations. These ways use such computational technologies as MPI (Message Passing Interface) [16] and DVM technology [17-22]. DVM technology [17-22] was elaborated in Keldysh Institute of Applied Mathematics RAS. DVM-system provides unified toolkit to develop parallel programs of scientific-technical calculations in $\mathrm{C}$ and Fortran. Unified parallel model is built in $\mathrm{C}$ and Fortran languages on the base of the constructions, that are "transparent" for standard compilers, that allows to have single version of the program for sequential and parallel execution. This way of code parallelizing allows one to save a lot of human resources for coding and debugging. For both types of parallel technologies special control interfaces for parameter optimization and analysis were designed $[7,8]$.

With the help of the constructed interfaces, a series of calculations were carried out, realizing the concept of a generalized numerical experiment for various classes of problems. The results of the calculations will be shown in the following sections. Both developed interfaces are very versatile. They can be applied to almost any software code for solving the CFD problem chosen as the base one.

According to [5-8], as a result of implementing the construction of a generalized numerical experiment and performing parallel calculations, we obtain a large data set representing a set of numerical solutions $F\left(x, y, z, t, A_{1}, \ldots, A_{N}\right)$ for each point $\left(A_{1}^{*}, \ldots, A_{N}^{*}\right)$ of the partition of the multidimensional volume of the defining parameters $\left(A_{1}, \ldots, A_{N}\right)$ of the problem under consideration. This volume in its original form is rather difficult to use, although its availability for further purposes is necessary. In order to get useful information from a calculated multidimensional data array, first of all we need to reduce its dimension. By lowering its dimension, we are able to apply the methods of visualization and visual analytics [23-26] in order to understand the internal structure of the array and to reveal hidden interdependencies between its defining factors. The revealed dependencies can be further approximated by geometric primitives in order to obtain a generalizing dependence, which will represent the solution of interest for a class of problems. Examples of the implementation of this approach are presented in [5-14].

Also, to reduce the dimension of a multidimensional array, methods of mapping into embedded manifolds of smaller dimension are very effective [27-29]. Among them, the most common method is the principal component method (PCA). The essence of the method consists in the transition from the initial coordinate system to the new orthogonal basis in the multidimensional space under consideration, whose axes are oriented along the directions of maximum dispersion. The possible scheme of working with an array in this case is the approximation by primitives of the data array in the space of the first three main components and the subsequent transition to the initial space of the determining parameters.

\section{Visualization in generalized numerical experiment}

The results of a generalized computational experiment, constructed as described in the previous sections, represent a multidimensional amount of data. One of the most effective tools to get the most information from this amount of data is a visual presentation. Visualization problems arising in a generalized computational experiment are described and systematized in dependence on the types of data in [10].

As a result of a generalized computational experiment, we obtain a numerical solution of the gas-dynamic problem $F\left(x, y, z, t, A_{1}^{*}, \ldots, A_{N}^{*}\right)$ for each point of space at any time and for each 
point of partition of the space of defining parameters $\left(A_{1}, \ldots, A_{N}\right)$. Here $F$ is a vector of gasdynamic functions, such as pressure, density, temperature, velocity components.

According to [10], one can define 3 types of visualization problems.

A) For each point of the partitioning of the spatial countable domain $(x, y, z)$ at the moment of time $t$ we have a whole set of solutions corresponding to each point of the partition of the space of defining parameters. From a practical point of view, the usefulness of such data is small, but such information can be very useful in assessing the contribution of the determining parameter $A_{i}$ to the total variance at each point of the computational domain and at any time moment. Here, for visualization at each point, it is advisable to use a classic spider diagram with normalization to the range of change of the determining parameter.

B) For each split point of the space of defining parameters $\left(A_{1}^{*}, \ldots, A_{N}^{*}\right)$ we have a calculated flow pattern in the selected region of space (the calculated region). This makes it possible to apply the entire rich set of scientific visualization methods and software [30, 31], developed earlier for gas dynamics problems. Possible types of informative visual representations include:

- visualization of scalar and vector fields in parallel sections of the computational domain and cross-sections;

- animation of scalar and vector fields in the constructed sections;

- construction of combined visual representations.

Such presentations can be created for each fixed point of a partition of the space of defining parameters $\left(A_{1}^{*}, \ldots, A_{N}^{*}\right)$. Here one has a new opportunity to construct the boundaries of change of the defining parameters of interest from $A_{i}^{\min }$ to $A_{i}^{\max }$ for each $A_{i}$. This can be useful, for example, in the visualization of separation zones, where it is possible to single out the limits of the change in the position of the separation zones, depending on the choice of the determining parameter. Such visual presentations for a particular class of problems can be created on the basis of already existing methods and algorithms implemented in many software systems, such as ParaView [32], VizIt [33], TecPlot [34].

C) From a practical point of view, when solving problems of computational gas dynamics, the primary interest to the user, as a rule, are valuable functionals calculated using the already computed gas-dynamic functions in the computational domain. The role of such a functional can be played by the total drag coefficient of a body in the flow or the friction drag coefficient for viscous problems. When analyzing the conditions for the emergence and decay of spacetime structures in a flow field, such a functional can be the characteristic time of the structure's existence.

According to [10], here we come to the classical tasks of visual analytics [23-28], where the goal is to obtain maximum information about the multidimensional data array and hidden relationships between its defining parameters. One of the possible approaches presented in $[9,35,36]$ is dimension reducing of the studied space of defining parameters to three, the visual representation of dependence obtained and approximation of this dependence using a set of geometric primitives. The purpose of this approach is to represent the dependence in question in an analytical form. If successful, this makes it possible to construct the obtained results of mathematical modeling in the form of a physical law for the class of problems in question.

\section{Some examples of generalized numerical experiment}

This section contains the examples of the generalized numerical experiment application to some practical problems. It is applied in some variations due to different aims for each class of problems.

The first example of generalized numerical experiment is devoted to the problem of tuning the properties of hybrid finite-difference schemes [12]. The paper [12] contains the description of developed program tool Burgers2. This program tool is intended for tuning and optimization of computational properties for hybrid finite-difference schemes applied to Burgers 
equation. One-dimensional model Burgers equation describes propagation of disturbances for dissipative medium. The equation has exact solution, so it is widely used for tuning-up of computational tools. Described program tool is based on combining of optimization problem solution and visual data presentation. Visual presentations of maximal error surface and error function are implemented as program tool features. Users have possibility of creating hybrid finite-difference schemes and analyzing computational properties for chosen grid template provided by program tool. Visual presentation of optimization problem solution allows finding of suitable weight coefficients for hybrid finite-difference scheme under consideration. The user is able to make simultaneous calculations varying weight coefficients in the scheme and viscosity coefficient in Burgers equation. The user can make the calculations simultaneously different sets of weight coefficients in accordance with the concept of generalized numerical experiment. Figure 1 presents the surface of absolute error for one of the hybrid scheme variants. The negative data area indicates where the oscillations occur.

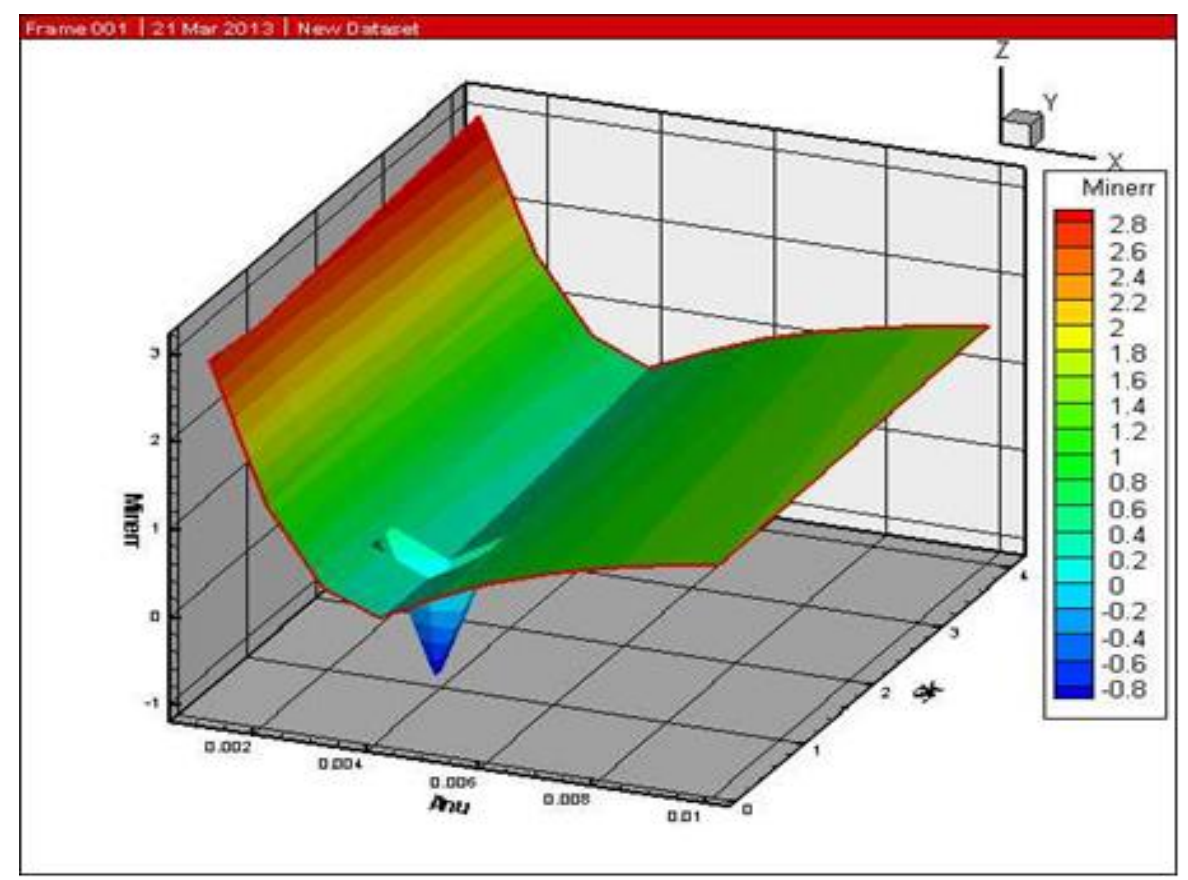

Fig. 1. Surface of absolute error for Burgers equation test [12].

The following example is also devoted to improving the computational properties of finitedifference schemes. The problem of mathematical modelling of the flow in the far wake behind the body is solved. In the general case, in a rectangular computational domain, a viscous compressible heat-conducting gas flow is considered, described by a complete system of timedependent Navier-Stokes equations. At the input boundary, the distributions of gas-dynamic parameters are given, obtained from calculations of the flow around an axisymmetric body and a portion of the track behind it. The main goal of the generalized computational method was to thoroughly study the properties of artificial viscosity incorporated in the hybrid difference scheme. For this purpose, we studied the properties of the weight coefficients of the hybrid scheme on the example of the problem of flow in the far wake and determined the limitations for the weight coefficients. In this task, the following defining parameters were varied, such as the steps of the grid decomposition in the $\mathrm{x}$ and $\mathrm{y}$ directions, the weighting coefficients of the difference scheme, the Reynolds number of the problem. As a result of the generalized computational experiment, a limit surface was constructed for the dependence of the weight coefficient on the other determining parameters of the problem. An example of the limiting surface is presented in figure 2 . When choosing the value of the weighting factor below the surface, in the numerical solution, non-physical oscillations arise, which can lead to the collapse of the solution. Such surfaces are constructed for non-viscous and viscous flow. In the case of viscous flow, laminar and turbulent regimes are considered. 


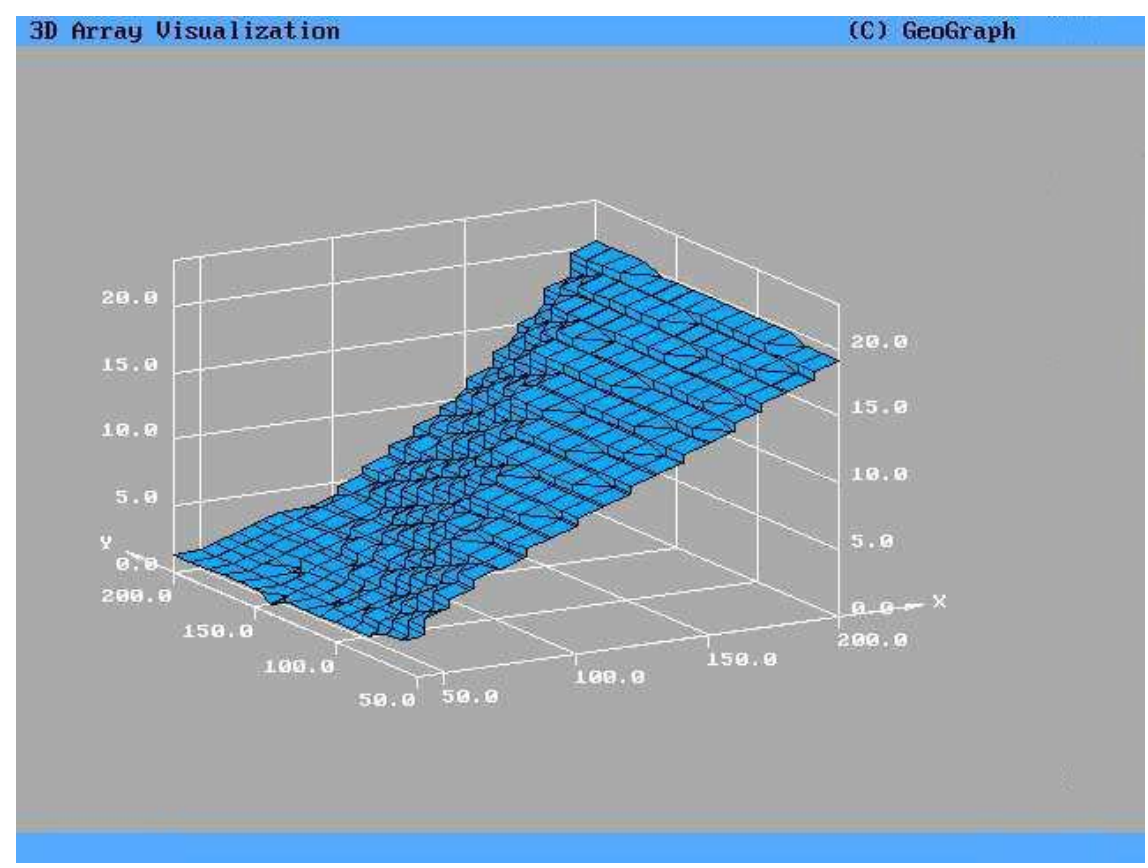

Fig. 2. Surface of absolute error for far wake problem.

The next example considers the problem of the evaluation of the accuracy for different numerical methods. The problem of inviscid compressible flow around a cone at zero angle of attack is used as a base one. The results obtained with the help of various OpenFOAM solvers are compared with the known numerical solution of the problem with the variation of cone angle and flow velocity [13]. Cone angle $\beta$ changes from $10^{\circ}$ to $35^{\circ}$ in steps of $5^{\circ}$. Mach number varies from 2 to 7 . For comparison, four solvers were selected from the OpenFOAM software package: RhoCentralFoam, SonicFoam, RhoPimpleFoam, RhoPimpleFoam. The results of such kind of numericsl experiment were presented as errors in the form of an analog of the L2 norm for all solvers. Figure 3 illustrates the results in a form of a change in deviation from the exact solution for pressure depending on the cone angle and the velocity for the solver rhoCentralFoam. Such changes were obtained for all solvers.

Figure 3 shows a multidimensional dataset for pressure obtained as a result of parametric calculations in the space of the first three principal components. Yellow shows the results for rhoCentralFoam solver, red for pisoCentralFoam, green for sonicFoam and blue for rhoPimpleFoam.

Figure 3 shows that the errors for rhoCentralFoam and for pisoCentralFoam can be roughly approximated by a plane reflecting the dependence of the error on the Mach number and cone angle. The results for sonicFoam and especially for rhoPimpleFoam are significantly separated from the results for the first two solvers due to their particular numerical characteristics. This methodical research can serve as a basis for selecting the OpenFoam solver for calculating the inviscid supersonic flow around the elongated bodies of rotation. The results of solvers comparison can also be useful for developers of OpenFoam software content. The results obtained made it possible to get a general idea of the calculation errors for all solvers. 


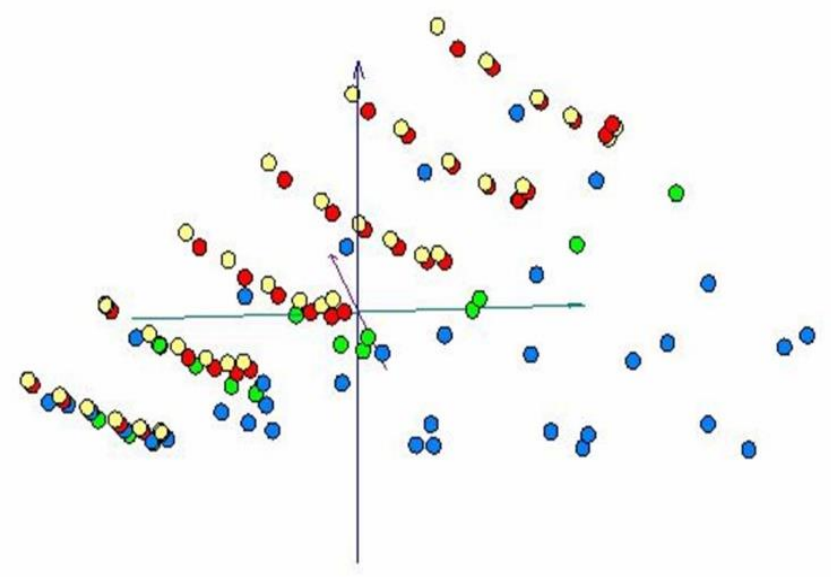

Fig. 3. Errors for different OpenFOAM solvers in the space of principal components.

The following example also focuses on the comparative evaluation of the accuracy of openFOAM solvers. The classical two-dimensional problem of a supersonic inviscid compressible flow falling on a flat plate at an angle of attack was considered as a test problem [14]. As a result, an oblique shock wave is formed before the start of the plate. The simulation results for the solvers considered in comparison are compared with the known exact solution. Calculations for all solvers participating in the comparison were carried out with the same setting of the parameters of the incident flow and angle of attack. Special attention was paid to QGDFoam solver, which has controlled dissipative properties. For this solver, within the framework of a general comparison, calculations were carried out with a variation of the parameter that allows controlling dissipative properties. The results of estimates of deviations from the exact solution in various norms for all solvers were obtained. Here, the implementation of a generalized computational experiment made it possible to quickly and efficiently obtain a comprehensive picture of the deviation of numerical solutions from the exact solution. Fig. 4 presents the distribution of pressure in the vicinity of the shock wave for different solvers in comparison with exact solution. Also, such distributions are presented for the QGDF solver when the smoothing parameter is changed.

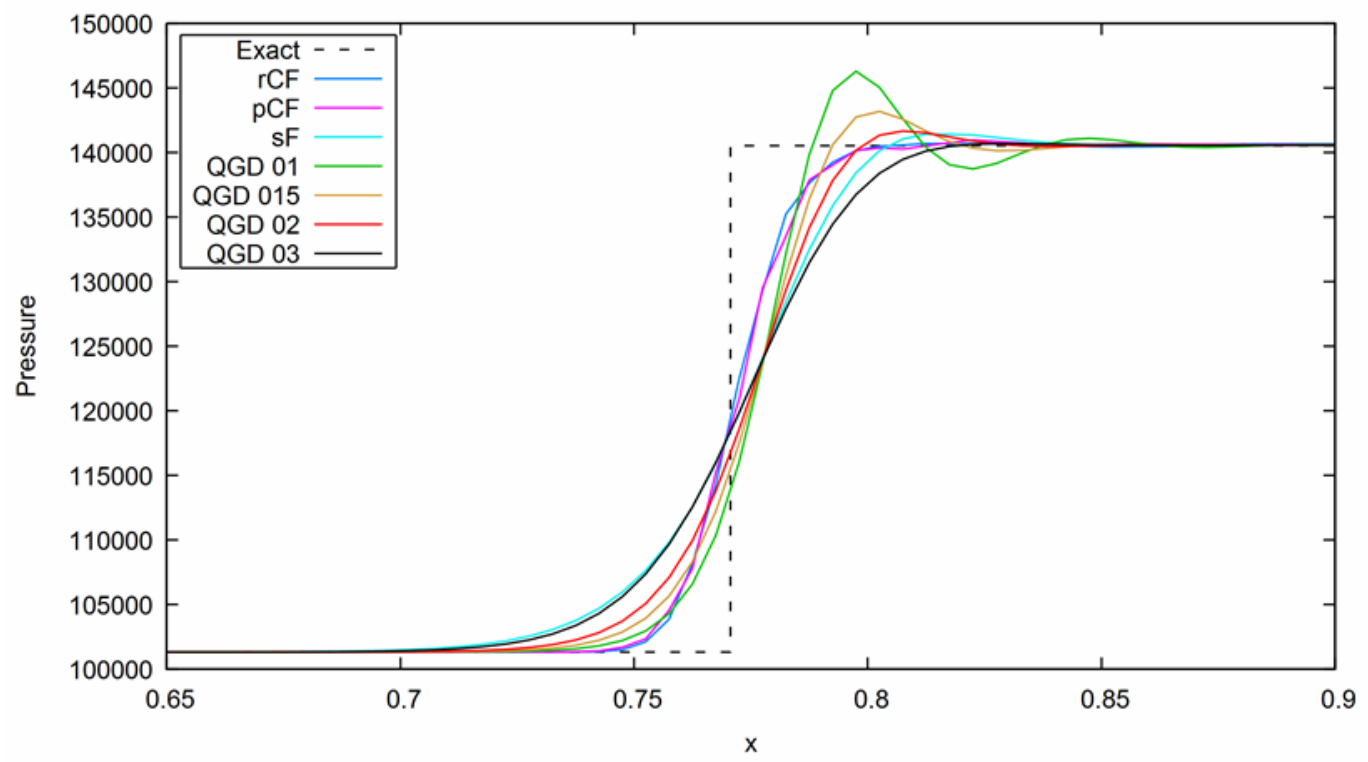

Fig. 4. The distribution of pressure in the vicinity of the shock wave for different solvers in comparison with exact solution [14]. 
The next example of application of general numerical experiment considers optimization problem. The example presents a search for optimal shape of three-dimensional blade assembly intended for power plant [37]. This experiment is based on developed computational technology for the computation of power loads on the $3 \mathrm{D}$ blade assembly of a power plant in a wind flow. The calculation for various combinations of the key geometric parameters of the assembly using parallel computations makes it possible to find the optimal shape of the assembly with respect to its power characteristics. A virtual experimental facility for simulating the flow around the power plant based on the solution of the Navier-Stokes equations was created. Computations aimed at determining the optimal shape of the blade assembly taking into account constraints on its design were carried out, and the results were thoroughly analyzed using the proposed optimization procedure. The solution of the optimization problem is based on the parameterization of the design using three key parameters. On the discrete set of values of these parameters, the maximums of two objective functions-the magnitude of the total aerodynamic force and the magnitude of the rotation torque-determining the lift-todrag ratio of the power plant are found. Figure 5 presents the shape of 3D blade assembly and pressure distribution on its surface.

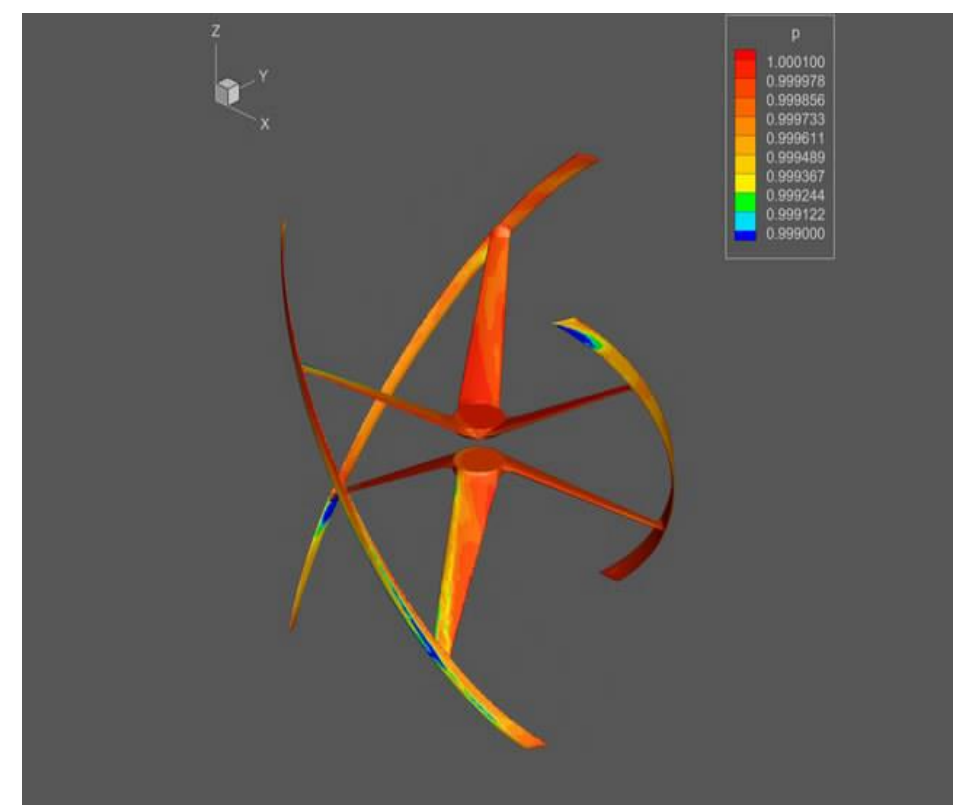

Fig. 5. Pressure distribution on the surface of 3D blade assembly [37].

The given examples show the applicability of the presented approach for a wide range of practical applications; therefore, the construction of a generalized computational experiment can be considered as a rather universal and useful approach.

\section{The perspectives of generalized computational exper- iment}

The above examples show the possibility of constructing a generalized computational experiment for various problems in the field of computational gas dynamics. Parametric studies can serve as such an experiment, where the basis is the ability to solve the direct problem of mathematical modeling. An example of an optimization analysis problem is given, where the generalized computational experiment is based on solving the inverse problem in an optimization statement. Thus, to create such an experiment is quite realistic for almost any mathematical modeling problem.

A separate area of application of a generalized computational experiment can be a comparative assessment of the accuracy of numerical methods. Similar attempts are presented in [3, $11,38]$ devoted to the problem of accuracy estimation with the help of the ensemble of solu- 
tions. According to $[3,11,38]$, if a researcher is able to calculate the same problem using several numerical methods with different computational properties, in particular, different approximation orders, then in some cases one can estimate the neighborhood of the approximate solution containing the exact solution (exact solution enclosure). If an ensemble of numerical solutions can be divided into clusters of "accurate" and "inaccurate" solutions, then the error ranking of values can be performed using an a posteriori analysis of the distances between the numerical solutions. This can serve as a computational proof of the existence of an exact solution in the case of nonlinear problems. This approach can be considered as perspective. Nevertheless, it has evident draw-back. For using of this approach one should have a set of solvers with different accuracy order.

\section{Conclusions}

The concept of generalized numerical experiment presented in the article has a wide range of possible applications. For the problems of computational fluid dynamics such an approach makes it possible to obtain a solution not only for one, separately taken, problem, but for a whole class of problems defined in a certain range of the complex of determining parameters. Practical implementation of the approach becomes possible with the use of parallel calculations in multitask mode. The results of calculations are multidimensional volumes of data that can be processed using data analysis tools and visual analytics. The application of these methods reveals hidden interdependencies between the determining parameters of the class of problems. Also, these methods allow in many cases to build the dependence of the valuable functional on the determining parameters, which makes it possible to further approximate it with geometric primitives and present it in an analytical form. The examples presented in the article illustrate generalized computational experiments for various types of tasks, such as improving the computational properties of difference schemes, finding the optimal body shape in a stream, a comparative assessment of the accuracy of algorithms. The approach is a synthesis of parallel computing, multi-dimensional data processing methods and visual analysis. The application of this approach makes it possible to increase the efficiency of research for a number of applied problems of mathematical modeling.

\section{References}

[1] Bondarev E.N., Dubasov V.T., Ryzhov Y.A., Svirschevsky S.B. and Semenchikov N.V. 1993 Aerigidromeckanika. (Moscow: Mashinostroenie) p 608 [In Russian]

[2] Kozlov L.V. "Experimental investigation of surface friction on a flat plate in a supersonic flow in the presence of heat transfer". 1963 Izvestiya AN SSSR, Mekhanika $i$ mashinostroenie 2 p11-20 [In Russian]

[3] Alekseev A.K., Bondarev A.E., Kuvshinnikov A.E. Verification on the Ensemble of Independent Numerical Solutions // Lecture Notes in Computer Science, Vol. 11540, pp. 315324, 2019. DOI: 10.1007/978-3-030-22750-0_25

[4] Andreev S.V. et al.: A Computational Technology for Constructing the Optimal Shape of a Power Plant Blade Assembly Taking into Account Structural Constraints. Programming and Computer Software, 43(6), 345-352 (2017). DOI: 10.1134/So361768817060020.

[5] Bondarev A.E. "Analysis of Space-Time Flow Structures by Optimization and Visualization Methods" // Transactions on Computational Science XIX, LNCS 7870, 158-168 (2013).

[6] Bondarev A.E. and Galaktionov V.A. "Parametric Optimizing Analysis of Unsteady Structures and Visualization of Multidimensional Data" // International Journal of Modeling, Simulation and Scientific Computing, V.04, suppo1, (2013) DOI 10.1142/S1793962313410043.

[7] Bondarev A.E. and Galaktionov V.A. "Analysis of Space-Time Structures Appearance for Non-Stationary CFD Problems" // Procedia Computer Science, 51, 1801-1810 (2015).

[8] Bondarev A.E. "Design and analysis of multidimensional parametrical solutions for timedependent CFD problems" // Mathematica Montisnigri, XXXIII, 58-68 (2015). 
[9] Bondarev A.E. On the Construction of the Generalized Numerical Experiment in Fluid Dynamics // Mathematica Montisnigri, Vol. XLII, 2018, p. 52-64.

[10] Bondarev A.E. On visualization problems in a generalized computational experiment (2019). Scientific Visualization 11.2: 156 - 162, DOI: 10.26583/sv.11.2.12

[11]Bondarev A.E. On the Estimation of the Accuracy of Numerical Solutions in CFD Problems // Lecture Notes in Computer Science, Vol. 11540, pp. 325-333, 2019. DOI: 10.1007/978-3-030-22750-0_26

[12]Bondarev, A.E. et al.: Design of program tool BURGERS2 for hybrid finite-difference schemes optimization and visualization. Scientific Visualization 5(1), 26-37 (2013).

[13]Bondarev A.E., Kuvshinnikov A.E. Analysis of the Accuracy of OpenFOAM Solvers for the Problem of Supersonic Flow Around a Cone // Lecture Notes in Computer Science 10862, pp. 221-230, 2018. https://doi.org/10.1007/978-3-319-93713-7 18

[14]Alekseev A.K., Bondarev A.E., Kuvshinnikov A.E. Comparative analysis of the accuracy of OpenFOAM solvers for the oblique shock wave problem // Matematica Montisnigri, 2019, vol. XLV, p. 95-105 DOI: 10.20948/mathmontis-2019-45-8

[15]Ivanov A. V., Khilkov S. A. "Aiwlib library as the instrument for creating numerical modeling applications", Scientific Visualization, 10(1), 110 - 127 (2018). DOI: 10.26583/sv.10.1.09

[16]Pacheco P., Programming Parallel with MPI. San Francisco, CA: Morgan Kaufmann, 1997.

[17]Aleksahin V. F., Bakhtin V. A., Zhukova O. F., Kolganov A. S., Krukov V. A., Ostrovskaya I. P., Podderugina N. V., Pritula M. N., Savitskaya O. A. "Parallelization of NAS parallel benchmarks for Intel Xeon Phi coprocessor in Fortran-DVMH language”, Vestn. YuUrGU. Ser. Vych. Matem. Inform., 4:4 (2015), 48-63

[18]Bakhtin V.A., Chetverushkin B.N., Kryukov V.A., Shil'nikov E.V., "Extension of the DVM parallel programming model for clusters with heterogeneous nodes", Doklady Mathematics, 84 (3), 879-881 (2013).

[19]Bakhtin V. A., Kolganov A. S., Krukov V. A., Podderyugina N. V., Pritula M. N. "Mapping DVMH-programs with regular dependencies onto clusters with GPU”, Vestn. YuUrGU. Ser. Vych. Matem. Inform., 2:4 (2013), 44-56

[20]Bakhtin V. A., Klinov M. S., Kolganov A. S., Krukov V. A., Podderyugina N. V., Pritula M. N. "Automatic mapping of Fortran programs onto clusters with graphics processing units”, Vestn. YuUrGU. Ser. Vych. Matem. Inform., 3:3 (2014), 86-96

[21]Bakhtin V. A., Zaharov D. A., Kolganov A. S., Krukov V. A., Podderyugina N. V., Pritula M. N. "Development of parallel applications using DVM-system", Vestn. YuUrGU. Ser. Vych. Matem. Inform., 8:1 (2019), 89-106

[22]DVM-system Available at: http://dvm-system.org/ (accessed 25 October 2019)

[23]Keim D., Kohlhammer J., Ellis G. and Mansmann F. (Eds.) Mastering the Information Age - Solving Problems with Visual Analytics, Eurographics Association, 2010.

[24]Kielman, J. and Thomas, J. (Guest Eds.) (2009). Special Issue: Foundations and Frontiers of Visual Analytics / Information Visualization, Volume 8, Number 4, p. 239-314.

[25]Thomas J. and Cook K. 2005 Illuminating the Path: Research and Development Agenda for Visual Analytics (IEEE-Press)

[26]Wong P. C., Thomas J. Visual Analytics // IEEE Computer Graphics and Applications. 2004. V. 24, N. 5. - P. 20-21.

[27]Gorban A, Kegl B, Wunsch D and Zinovyev A 2007 Principal Manifolds for Data Visualisation and Dimension Reduction (Berlin - Heidelberg - New York: Springer)

[28]Gorban A.N., Zinovyev A. "Principal manifolds and graphs in practice: from molecular biology to dynamical systems", International Journal of Neural Systems, Vol. 20, No. 3 (2010). Pp. 219-232.

[29]Zinovyev A 2000 Vizualization of multidimensional data (Krasnoyarsk: Krasnoyarsk Univ.) 
[30]Bondarev A. E. and Bondarev E. N. Visualization Functions in Computational Fluid Dynamics / Obshcheros. NauchnoTekhn. Zh. Polet (Mashinostr, Moscow, 2000), No. 10, pp. 53-60 [in Russian].

[31]Bondarev A.E., Galaktionov V.A., Chechetkin V. M. Analysis of the Development Concepts and Methods of Visual Data Representation in Computational Physics / Computational Mathematics and Mathematical Physics, 2011, Vol. 51, No. 4, pp. 624-636.

[32]ParaView system Available at: https://www.paraview.org/ (accessed 25 October 2019)

[33]VizIt Available at: https://sourceforge.net/projects/visit/ (accessed 25 October 2019)

[34]TecPlot Available at: https://www.tecplot.com/ (accessed 25 October 2019)

[35]Bondarev A.E., Galaktionov V.A. Multidimensional data analysis and visualization for time-dependent CFD problems / Programming and Computer Software. 2015. Vol. 41. №. 5. P. 247-252. DOI: 10.1134/So361768815050023

[36]Bondarev A.E., Bondarenko A.V., Galaktionov V.A. (2018) Visual analysis procedures for multidimensional data. Scientific Visualization 10.4: 109 - 122, DOI: 10.26583/sv.10.4.09

[37]Andreev S.V. et al.: A Computational Technology for Constructing the Optimal Shape of a Power Plant Blade Assembly Taking into Account Structural Constraints. Programming and Computer Software, 43(6), 345-352 (2017). DOI: 10.1134/So361768817060020.

[38]Alekseev A.K., Bondarev A.E. Estimation of the Distance between True and Numerical Solutions. Computational mathematics and mathematical physics, 2019, T. 59, № 6, c. 857-863 DOI: 10.1134/So965542519060034 